\title{
L'imaginaire géographique dans Alma de Jean- Marie Le Clézio Étude géocritique
}

\author{
Dr. Kamel Mounir \\ Faculté des Lettres -Menoufeya
}

\section{Résumé}

Cet article se propose d'aborder l'imaginaire géographique dans Alma de Jean-Marie Le Clezio. Ce roman se situe dans l'évolution du discours narratif leclézien et se caractérise par une spatialisation croissante des formes narratives liées aux îles lecléziennes. L'étude du texte leclézien nous amène à mettre au premier plan l'espace géographique en tant que thème essentiel qui exprime la spécificité de la pratique de l'écriture créative dans Alma de Le Clézio. Pour essayer de mieux cerner le rapport entre espace et littérature, nous allons nous focaliser, à travers une approche géocritique, sur les spécificités de l'espace qui se considère comme un moyen de communication mise en contexte en fonction des deux univers : littéraire et géographique. D'un point de vue littéraire, le roman d'Alma de Jean-Marie Le Clézio (2017) suppose de faire passer à la géographie littéraire les processus qui concourent à l'analyse du fait littéraire dans l'espace. L'examen de cette géographie littéraire peut porter sur trois thèmes distincts : les paysages de l'île Maurice, la centralité de la famille, et enfin la description de la tyrannie diabolique.

Mots-clés: imaginaire géographique, géographie littéraire, géocritique, discours narratif, création littéraire. 


\section{التَّيل الجغراني في رواية ألما عند جون هاري لو كليزيو دراسة جغرافية نقدية}

\section{د. كاهل هممد هنير همهد كاهل هوسى \\ مدرس الأدب الفرنسي - كلية الآداب بالمنوفية - جامعة المنوفية هواسية}

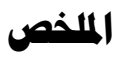

يقترح هذا المقال استعراض التَّيل الجغرافي في رواية ألما عند جون ماري

لو كليزيو. تدخل هذه الرواية ضمن تطور الخطاب السردي عند لو كليزيو وتتميز بإضفاء الطابع الدكاني السردي المرتبط بالجزر التي يتطرق اليها لو كليه كليزيو.

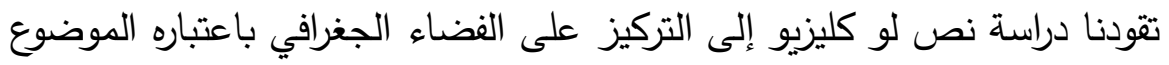

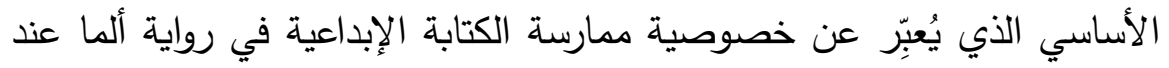
لو كليزيو. ولمحاولة فهم العلاقة بين الفضاء المكاني والفضاء الأدبي بشكل

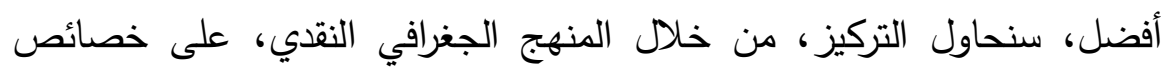

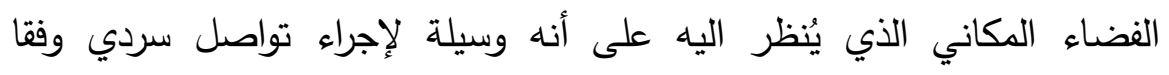

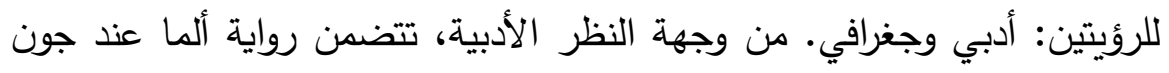

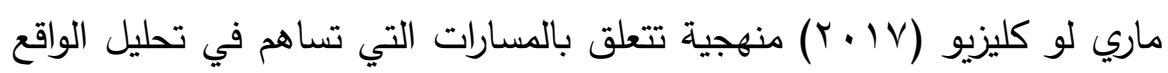

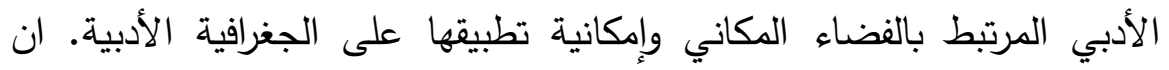

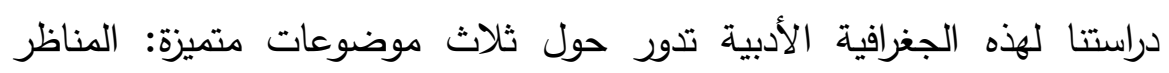
الطبيعية في جزيرة موري، الدور المركزي للأسرة، وأخيرا، وصف الأفئ الاستبداد الشيطاني.

الكلمات المفتاحية: التَّيل الجغرافي، الجغرافية الأدبية، الجغرافية النقدية،

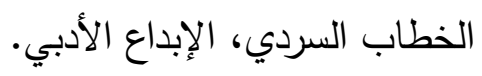


L'imaginaire géographique dans Alma de Jean-Marie Le Clézio Étude géocritique

Dr. Kamel Mounir

مجلة وادي النيل للار اسات والبحوث الإسانية والاجتماعية والتربوية (مجلة علمية محكمة)

\section{L'imaginaire géographique dans Alma de Jean-Marie Le Clézio $^{(1)}$ : Étude géocritique ${ }^{(2)}$}

\section{Introduction}

Jean-Marie Le Clézio empreinte à la géographie la méthode de la description du vécu. Ce procédé consiste à s'immerger dans l'imaginaire géographique. L'auteur raconte les évènements qu'il observe dans le lieu qu'il fréquente, le lieu qui lui est intime, celle de l'île Maurice qui reste le centre de son imaginaire littéraire. Sachant que celui-ci joue un rôle crucial dans le développement des évènements. On peut trouver cette lucidité dans les descriptions saisissantes des lieux.

« Les paysages décrits par Le Clézio sont à la fois précis et imprécis. Géographiquement fixés et assez

1. «En l'espace de vingt romans, de six recueils de nouvelles et d'un recueil de contes, Jean-Marie Gustave Le Clézio est devenu l'un des écrivains français les plus lus, les plus aimés des lecteurs et les plus consacrés par les instances de légitimation. Lauréat de neuf prix littéraires couronnés par le prix Nobel en 2008 (13ème Prix Nobel français), l'originaire de l'Île Maurice a été désigné en 1994 par les lecteurs du Magazine Lire comme le «plus grand écrivain francophone vivant » à côté des écrivains tels que Nathalie Sarraute, Claude Simon, Françoise Sagan, Michel Tournier ou encore Julien Gracq. " «Problématiques écologiques dans les écrits de Jean-Marie Gustave Le Clézio» Université de Dschang, Cameroun - date limite 15 mars 2019, Équipe de Recherche en Littérature Comparée (ERLIC)

2. « La géocritique promue par Bertrand Westphal semblerait pouvoir converger avec les préoccupations d'une géo-littérature, puisque sa démarche est "géocentrée », l'accent étant placé " davantage sur l'espace observé que sur l'observateur saisi dans sa spécificité. " Rosemberg Muriel, «La spatialité littéraire au prisme de la géographie », L'Espace géographique, 2016/4 (Tome 45), p. 289-294. DOI : $10.3917 /$ eg.454.0289. 
مجلة وادي النيل للار اسات والبحوث الإسانية والاجتماعية والتربوية (مجلة علمية محكمة)

(ISSN : 2536 - 9555)

vastes pour évoquer à ceux qui en lisent la description, d'autres mondes ou d'autres lieux. Toutes les mers sont la mer, tous les rivages lointains sont le rivage, tous les ciels, le ciel. » (Gazier, 2010 : pp. 21-28)

Omniprésent dans les écrits de Jean-Marie Le Clézio, l'imaginaire géographique fait, généralement, partie du langage romanesque de notre romancier. Le choix de l'imaginaire géographique n'est jamais fortuit, l'auteur cerne le contexte dans lequel se déroulent les évènements historiques d'Alma $^{(3)}$ et se penche sur les lieux qui sont parsemés de diverses sortes d'histoires vivantes. Comme toute créativité littéraire, le roman de Jean-Marie Le Clézio cherche à étudier les spécificités de l'espace qui se considère comme un moyen de communication mise en contexte en fonction de deux univers : littéraire et géographique.

Alma de Jean-Marie Le Clézio permet au lecteur d'interpréter la réalité humaine du passé historique, sachant que celle-ci est située dans un paysage spatial précis. Par souci de brosser un portrait de ce paysage, Alma de Jean-Marie Le

3. Alma, le dernier roman de Jean-Marie Le Clézio, est un roman construit sur le séjour de Jérémie sur l'île Maurice qui se transforme en une investigation sur l'histoire de sa famille. Cette transformation l'a conduit à évoquer la question de l'esclavage et du racisme. Cette histoire de famille au passé esclavagiste amène le lecteur à découvrir l'ile Maurice à travers deux personnages Jérémie et Dodo qui parcourent les deux axes du roman; l'un court vers son île pour chercher la vérité et l'autre quitte ce lieu pour se retrouver parmi les exclus. Ce roman met donc en relief une histoire de deux destins croisés : "Dans le jardin de la Maison Blanche le soleil d'hiver passe sur mon visage, bientôt le soleil va s'éteindre, chaque soir le ciel devient jaune d'or. Je suis dans mon île, ce n'est pas l'île des méchants, les Armando, Robinet de Bosses, Escalier, ce n'est pas l'île de Missié Kestrel ou Missié Zan, Missié Hanson, Monique ou Véronique, c'est Alma, mon Alma, Alma des champs et des ruisseaux, des mares et des bois noirs, Alma dans mon cœur, Alma dans mon ventre. » (Le Clézio : p. 260)

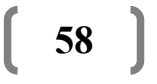




\section{L'imaginaire géographique dans Alma de Jean-Marie Le Clézio Étude géocritique}

Dr. Kamel Mounir

مجلة وادي النيل للاراسات والبحوث الإنسانية والاجتماعية والتربوية (مجلة علمية محكمة)

Clézio nous convie à faire le point sur la géographie mauricienne.

D'un point de vue littéraire, Alma de Jean-Marie Le Clézio suppose de faire passer à la géographie littéraire les processus qui concourent à l'analyse du fait littéraire dans l'espace. L'examen de cette géographie littéraire peut porter sur trois thèmes distincts: les paysages de l'île Maurice, la centralité de la famille, et enfin l'écriture de l'espace. En fait, notre approche d'analyse géocritique ${ }^{(4)}$ permet de décrire un lieu dans lequel le romancier parle des paysages qui ont de l'influence sur lui. Cet approche à la fois descriptive et critique nous permettra de nous rendre compte des événements qui se passent dans des lieux précis.

4. « La géo-critique n'aura pas à emprunter cette voie, car elle se fonde sur le postulat inverse : les espaces humains ne deviennent pas imaginaires en intégrant la littérature ; c'est la littérature qui leur octroie une dimension imaginaire, ou mieux : qui traduit leur dimension imaginaire intrinsèque en les introduisant dans un réseau intertextuel. La géo-critique, en effet, se propose d'étudier non pas seulement une relation unilatérale (espace-littérature), mais une véritable dialectique (espace-littérature-espace) qui implique que l'espace se transforme à son tour en fonction du texte qui, antérieurement, l'avait assimilé. Les relations entre littérature et espaces humains ne sont donc pas figées, mais parfaitement dynamiques. L'espace transposé en littérature influe sur la représentation de l'espace dit réel (référentiel), sur cet espacesouche dont il activera certaines virtualités ignorées jusque-là, ou réorientera la lecture. Si la ville était livre, ou même palimpseste, il serait normal qu'elle fît l'objet d'une esthétique de la réception. On pourrait éventuellement lire une ville en suivant les indications de Gérard Genette ou de Hans Robert Jauss. » Bertrand WESTPHAL, "Pour une approche géo-critique des textes", in La Géo-critique mode d'emploi, PULIM : Limoges, coll. «Espaces Humains », n0, 2000, pp.9-40., consulté le 08 Octobre 2021. 
مجلة وادي النيل للار اسات والبحوث الإسانية والاجتماعية والتربوية (مجلة علمية محكمة)

(ISSN : 2536 - 9555)

Nous nous attacherons, dans cette perspective géocritique, à étudier quelques extraits d'Alma de Jean-Marie Le Clézio. Le choix de notre article consiste à montrer l'importance des paysages décrits par Le Clézio dans l'espace maritime mauricienne. Sachant que la géo-critique représente un dialogue entre la géographie et la littérature, c'est la raison pour laquelle nous essaierons d'analyser l'espace géographique qui devrait être un critère essentiel dans notre texte romanesque.

\section{Sur les traces de l'histoire vivante de l'île Maurice ${ }^{(5)}$}

Le choix de l'utilisation de quelques phrases marquantes de l'écriture leclézienne laisse apparaître une volonté chez notre romancier de faire entrer le lecteur dans l'espace de l'île Maurice.

En effet, le point que nous voulons approfondir dans cet article relève de l'analyse du texte littéraire : ce sont des expressions marquantes et des formes expressives. Le passage suivant s'ouvre sur une expression intentionnellement marquée : «la vieille carte de Maurice.» En voici un exemple représentatif :

«J'ai gardé pourtant la vieille carte de Maurice au 1/25 000, imprimée par Descures, datée de 1875, sur une toile jaunie enroulée autour d'une canne de bambou. On

5. «Découverte par les Portugais en 1507, puis occupée par les Hollandais de 1598 à 1710, Maurice devint la possession de la Compagnie des Indes en 1715, et du roi de France en 1767, qui l'a baptisée Île de France. Conquise en 1810 par les Anglais, et après s'être fait reconnaitre par le traité de Paris en 1814, les Anglais laissèrent aux colons français l'usage de leur langue et du code civil. » L'HISTOIRE DE L'ÎLE MAURICE, http://ilemauricehotels.com/ile-mauricehistoire.html

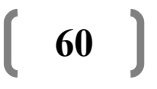


L'imaginaire géographique dans Alma de Jean-Marie Le Clézio Étude géocritique

Dr. Kamel Mounir

مجلة وادي النيل للاراسات والبحوث الإنسانية والاجتماعية والتربوية (مجلة علمية محكمة)

y voit toutes les parcelles avec les noms de leurs propriétaires, et les anciennes sucreries. Bien sûr j'ai vu Alma avec le nom des Felsen, mais ce n'est pas pour ça que j'ai voulu la garder. Non par nostalgie, mais parce que le découpage précis et les hachures du relief pouvaient me guider dans ma quête de l'oiseau disparu, parce que certains de ces noms et certains de ces lieux étaient les uniques témoins de cette histoire.» (Le Clézio, p. 25.)

Comme son nom l'indique la carte de Maurice représente un espace géographique, si ce n'est une carte imaginaire. ${ }^{(6)}$ Ainsi, cette carte entre dans cet espace géographique et imaginaire au sein duquel l'histoire humaine tout entière se retrouve. La description de cette carte est une spatialité littéraire leclézienne au prisme de la géographie avec laquelle les personnages construisent leurs espaces imaginaire et perçoivent le monde qui les entoure.

Dans l'exemple suivant, c'est la description de la carte qui va impliquer un certain indice descriptif permettant de savoir comment l'auteur a procédé pour

${ }^{6}$ - « Représenter l'Europe avec l'Espagne pour tête n'est pas un hasard. Il s'agit de montrer le pouvoir de Charles Quint sur l'Europe et la cohérence d'un continent que l'on veut, selon le concepteur de cette carte, placée sous l'autorité du Saint-Empire romain germanique (...). On voit apparaître des contrepropositions à cette carte, d'autres interprétations, dont une visiblement protestante qui montre le Saint-Empire romain germanique démembré. La reine Isabelle est représentée avec une robe, qui constitue le continent européen. La robe se fragmente, laissant apparaître sa poitrine nue, ce qui n'est pas respectueux. » Xavier Mauduit et Laurent Baridon, SÉRIE L'HISTOIRE À LA CARTE (4 ÉPISODES) Épisode 4 : Cartographier l'imaginaire 
مجلة وادي النيل للاراسات والبحوث الإنسانية والاجتماعية والتربوية (مجلة علمية محكمة)

(ISSN : 2536 - 9555)

faire entrer son lecteur dans l'ambiance de son texte romanesque : «sur une toile jaunie enroulée autour d'une canne de bambou. On y voit toutes les parcelles avec les noms de leurs propriétaires, et les anciennes sucreries.»

Une partie de la réussite de l'écriture leclézienne ${ }^{7}$ repose sur son aptitude à manipuler l'art de la description. Le Clézio se sert de cet art pour communiquer aux noms des autres avec les autres. Voici comment il est allé à la quête ${ }^{(8)}$ des «disparus de différentes espèces car les effets de la colonisation ont atteint non seulement la famille des narrateurs et d'autres habitants de l'île mais également les écosystèmes insulaires. " (9) Examinons le passage suivant:

7. L'ile Maurice est un voyage vers les ancêtres pour chercher les traces de la famille. Ce voyage se transforme contre la modernisation de l'ile et aussi contre les vices humains : "J'ai voulu aussi recoller les morceaux d'une histoire brisée, celle des Felsen de l'île, à présent aussi éteints que l'oiseau lui-même, dead as a dodo. Peut-être était-ce de la vanité, ce sentiment d'appartenir à une tribu en train de disparaître, d'être le témoin, le signal faible et vacillant d'une autre ère, d'une autre culture, autour des derniers survivants le monde est en train de changer, ne dit-on pas avec une certaine arrogance, à chaque génération, que rien ne sera plus comme avant ?» (Le Clézio, p. 266) Dans cette perspective, nous constatons que «l'écriture pour Jean Marie Le Clézio serait un voyage à rebours vers un monde primordial, une glaise primitive dont la conscience nous à exilé, que les concepts et les classifications ont fait se dissoudre. Elle serait retour et transcendance.» Labbé Michelle. L'Écart romanesque, paris, Ed.L'Harmattan, 1999. P.72

${ }^{8}$ - Le Clézio consacre des pages poignantes à la disparition du dodo, ce gros pigeon de l'île Maurice qui s'est éteint à la fin du XVIIe siècle : Dormez, gros oiseaux, gros dodos, glissez-vous vers les songes, fermez vos yeux au monde et entrez dans la préhistoire, vous, les derniers habitants d'une terre qui n'a pas connu les hommes !» Elisabeth Philippe, Disparition des oiseaux : les écrivains l'avaient prédit

9 - NATALIA NIELIPOWICZ, La restitution des voix disparues dans Alma de J. M. G. Le Clézio 


\section{L'imaginaire géographique dans Alma de Jean-Marie Le Clézio Étude géocritique}

Dr. Kamel Mounir

مجلة وادي النيل للاراسات والبحوث الإنسانية والاجتماعية و التربوية (مجلة علمية محكمة)

«Bien sûr j'ai vu Alma avec le nom des Felsen, mais ce n'est pas pour ça que j'ai voulu la garder. Non par nostalgie, mais parce que le découpage précis et les hachures du relief pouvaient me guider dans ma quête de l'oiseau disparu, parce que certains de ces noms et certains de ces lieux étaient les uniques témoins de cette histoire.»》

À ce jour cette histoire hybride se présente comme un modèle d'écriture embrassant avec divers espaces géographiques. Le Clézio n'a de cesse de mêler faits historiques et géographiques pour interroger le passé et porter un regard critique sur le présent. Il s'interroge sur l'histoire des «oiseaux disparus» pour enquêter sur les zones grises de l'histoire des êtres vivantes perdues. ${ }^{(10)}$ Dans le cadre de cette partie sur les traces de l'histoire vivante, nous nous sommes arrêtés sur les séquences qui concernent deux mondes : le monde animal et le monde végétal. La relation plante-animal est fort connue chez Le Clézio. En examinant le passage suivant, nous remarquons qu'il donne lieu à une image de la plante souffrante :

" ...c'est ça qu'il mangeait, ton oiseau dodo. On a prétendu qu'après la mort du dodo les tambalacoques ne pouvaient pas survivre, parce qu'il était le seul à pouvoir

10 - « Parlant d'Alma, son dernier roman, JMG Le Clézio lie le destin du dodo, cet oiseau mythique de l'île Maurice, aujourd'hui disparu, à un arbre, le tambalacoque (Sideroxylon grandiflorum ou Calvaria major). L'oiseau avalait et disséminait les graines de l'arbre. Depuis la disparition du dodo, l'arbre ne pousse plus à Maurice. Il était familièrement appelé au temps des Anglais, "the dodo-tree ». Christophe, Le tambalacoque (Alma Consult aime Alma de JMG Le Clézio), 23 octobre 2017 
مجلة وادي النيل للار اسات والبحوث الإنسانية والاجتماعية والتربوية (مجلة علمية محكمة)

(ISSN : 2536 - 9555)

digérer l'écorce de la graine, à la briser avec sa pierre à gésier, mais regarde, celui-ci est tout jeune, il prouve que l'arbre va continuer à vivre... »(Le Clézio, p. 104.)

Ce dernier point mérite que nous nous y arrêtions : la disparition des tambalacoques nous incite à réfléchir sur le mutualisme entre le dodo mauricien et le tambalacoque. Deux questions se posent : pourquoi les tambalacoques sont-elles disparues sur la terre Mauricienne? Comment la mort de dodo peut-il empêcher les tambalacoques de se mettre à pousser sur l'ile Maurice?

À la souffrance de la disparition de cet arbre s'est ajoutée celle de la mort de dodo. Est-il possible de surmonter la souffrance des mauriciennes qui est liée à la disparition de ces deux êtres vivants ? Le Clézio fait vivre le lecteur dans la tristesse et empêche la joie de s'extérioriser.

Nous terminons cette partie sur l'idée que notre romancier parle de la disparition des tambalacoques et de la mort de dodo en tant que un événement historique. L'exemple de la mort est vu comme quelque chose de personnel, sachant que cet événement historique se déroule souvent dans la stricte intimité familiale.

\section{La famille comme référence primordiale de l'existence humaine}

Aux yeux de Le Clézio, la famille est une source primordiale d'amour et de bonheur. L'auteur cherche tout le temps les secrets de famille qui ont des effets sur ceux qui ne les connaissent pas. A travers l'exil qui se considère comme 


\section{L'imaginaire géographique dans Alma de Jean-Marie Le Clézio Étude géocritique}

Dr. Kamel Mounir

مجلة وادي النيل للاراسات والبحوث الإنسانية والاجتماعية و التربوية (مجلة علمية محكمة)

l'écho de la terre perdue et surtout de la culpabilité qui tourmente les descendants d'esclavagistes, Le Clézio, dans son roman Alma, cherche les origines de sa famille mauricienne à travers l'enquête menée par ses personnages qui ont découvert dans l'ile Maurice les secrets, les habitants et aussi le passé insaisissable. Citons à titre d'exemple :

«Jeanne Tobie est une femme sans âge, petite et sèche, les yeux noirs, les cheveux coupés court d'un gris sale. Sa peau est ridée, tannée, tachée par le soleil. Elle sort me parler directement. Elle se tient debout devant moi, les mains dans les poches de son pantalon trop large pour elle. «Vous êtes qui ? J'hésite à lui répondre, elle redit, avec impatience : "Qui vous êtes ? Votre nom ? »Mon prénom ne lui dit rien, je mentionne le nom de ma mère, Alison O'Connor, et celui de mon père, Alexandre Felsen. J'ai connu un Felsen autrefois, un fou qui circulait partout vêtu comme un épouvantail. Un perdi bande, comme on dit à Maurice. Et puis il a disparu, on ne sait où. Un perdi bande, quelqu'un qui a perdu sa bande, sa famille, sans amis, un clochard. " (Le Clézio, pp. 69-70.)

La description minutieuse que donne Le Clézio au portrait physique de Jeanne Tobie nous laisse appréhender la thématique de son corps sans âge et ses yeux noirs. Si le thème de ses cheveux coupés court d'un gris sale correspond au thème de sa peau plein de ride, la manière de se tenir debout et de mettre les mains dans les poches rime avec le thème de sa façon de poser une question : «Vous êtes qui ?»

Les références à des réponses expressives font en sorte que Alma de J. M. G. Le Clézio fonctionne comme un code socio-familial : " Mon prénom ne lui dit rien, je 
mentionne le nom de ma mère, Alison O'Connor, et celui de mon père, Alexandre Felsen. " Le Clézio a été influencé par les origines familiales qui pèsent sur lui. Il est parti à la découverte de son père en ile Maurice.

Le Clézio excelle dans la description de tout ce qui rapporte à son père qui se bat peut être pour s'enfoncer dans l'ignorance : « J'ai connu un Felsen autrefois, un fou qui circulait partout vêtu comme un épouvantail. Un perdi bande, comme on dit à Maurice. Et puis il a disparu, on ne sait où. Un perdi bande, quelqu'un qui a perdu sa bande, sa famille, sans amis, un clochard. » Dans une perspective critique, nous pouvons nous en tenir à la conclusion que Le Clézio tend à donner des traits reflétant certains éléments qui déterminent les aspects physiques et sentimentales de l'existence son père. L'absurdité de la vie en ile Maurice oblige le père à quitter de la maison familiale et à vivre en vagabondage.

En fait, notre roman se donne à lire comme l'œuvre clé de l'image de la vie familiale et de la fraternité qui se sont reflétées dans bon nombre de passages, tels que l'extrait suivant :

« Tu vois, Jérémie, quand ton père est parti d'ici, j'ai eu l'impression que mon petit frère s'en allait, il a promis de m'écrire, mais une fois en France il a tout oublié, juste une fois quand je me suis mariée il m'a envoyé une carte, congratulation, même pas du français, et sa signature, plus rien. J'avais son adresse, mais je ne lui ai pas écrit moi non plus. J'ai pensé que tout ça était fini. Et c'est bien fini, n'est-ce pas ? Il ne reste plus rien de ce temps-là. Mon mari est mort, et nous étions ruinés, mes enfants sont allés vivre ailleurs, un en France, l'autre en Australie, tous mes petits-enfants sont ailleurs, en Suisse, au Sud-Afrique. Ils font des études, 


\section{L'imaginaire géographique dans Alma de Jean-Marie Le Clézio Étude géocritique}

Dr. Kamel Mounir

مجلة وادي النيل للاراسات والبحوث الإنسانية والاجتماعية و التريوية (مجلة علمية محكمة)

ils ne viennent qu'une fois par an, et ils vont plutôt à la mer, Moka ça ne les intéresse pas, tu vois où je vis ? Ils téléphonent au Chinois, mais c'est juste pour savoir si je suis encore vivante. Alors toi qui viens me voir, je ne peux pas te dire, c'est mon histoire qui revient, Alma, les champs de cannes, le ruisseau, l'étang, tout ça qui n'existe plus, vois ce qu'il en reste ! » (Le Clézio, p.79.)

Force est de constater que Le Clézio contribue à l'élaboration d'une description globale du mythe familial. Avec ce thème nous entrons dans l'univers d'une famille tourmentée : «j'ai eu l'impression que mon petit frère s'en allait, il a promis de m'écrire, mais une fois en France il a tout oublié, juste une fois quand je me suis mariée il m'a envoyé une carte, congratulation, même pas du français, et sa signature, plus rien $\gg$.

Ces traits descriptifs contribuent à l'illustration du rôle thématique qu'assume le petit frère. Il s'en va, laissant derrière soi sa famille au désespoir. La question qui se pose est la suivante : comment réagit le lecteur face à la négligence de ce petit frère de sa langue maternelle ? Pour répondre à cette question, il suffit de souligner que Le Clézio par l'emploi du vocable «congratulation » semble attristé de constater que ce petit frère suit la voie de cette perte morale. ${ }^{(11)}$

Le Clézio s'interroger sur la parenté entre le déchirement familial et la relation humaine brisée où le profit passe avant la morale. Le roman pose une série de relations humaines déchirées dans un cadre familial : «Il ne reste plus

${ }^{11}$ - C'est un devoir d'aimer sa langue maternelle. La négliger est un signe de décadence morale.” De Docteur Motta, le figaro, France. 
(ISSN : 2536 - 9555)

rien de ce temps-là. Mon mari est mort, et nous étions ruinés, mes enfants sont allés vivre ailleurs, un en France, l'autre en Australie, tous mes petits-enfants sont ailleurs, en Suisse, au Sud-Afrique. Ils font des études, ils ne viennent qu'une fois par an, et ils vont plutôt à la mer, Moka ça ne les intéresse pas, tu vois où je vis? Ils téléphonent au Chinois, mais c'est juste pour savoir si je suis encore vivante. »

Dans l'espace familial de notre roman, nous assistons à une douleur morale. L'auteur murmure d'un ton désespéré : «Alors toi qui viens me voir, je ne peux pas te dire, c'est mon histoire qui revient, Alma, les champs de cannes, le ruisseau, l'étang, tout ça qui n'existe plus, vois ce qu'il en reste ! $\gg \mathrm{Ce}$ n'est pas un hasard si la trame de la désespérance est posée au même niveau que les problèmes socio-familiaux dans Alma de Jean-Marie Le Clézio. Ce qui semble clair, c'est que la famille déchirée dans l'écriture leclézienne se veut de façon de plus en plus manifeste comme une critique de la société mauricienne.

\section{L'art de décrire la tyrannie diabolique selon Le Clézio}

Nous prenons comme point de départ un article marquant publié par le site internet Enviedécrire, «L'écriture selon Jean-Marie Gustave Le Clézio ». Cet article suppose un modèle d'écriture qui permet d'instaurer un lien entre la pensée et le comportement des êtres humains :

«Mon écriture est devenue plus défensive, comme une voie de fuite de la société occidentale, violente et artificielle où tout le monde rencontre la mort, l'envahissement, l'asservissement des objets et l'agression de la vie. Je cherche à retranscrire le monde, à faire en sorte que la pensée humaine corresponde avec le texte. A mon avis, c'est la tâche de l'auteur : 


\section{L'imaginaire géographique dans Alma de Jean-Marie Le Clézio Étude géocritique}

Dr. Kamel Mounir

مجلة وادي النيل للاراسات والبحوث الإنسانية والاجتماعية و التريوية (مجلة علمية محكمة)

retranscrire les expériences et déchiffrer ce que cela dit des comportements humains. ॥ ${ }^{(12)}$

Quant à l'art de cette écriture chez Le Clezio, il ressemble à une danse de mots. ${ }^{(13)}$ Cet art, qui s'empare du roman leclézien, est fortement enraciné dans l'histoire et la géographie mauricienne. En effet, Le Clezio sait que les envahisseurs sont là et qu'ils ont pris forme inhumaine. Il dresse le portrait de ces envahisseurs. Il parle de ses comportements cruels pendant les opérations militaires de la conquête à travers une description minutieuse de leurs physiques. Pour illustrer ce point, citons un passage qui dépeint les caractéristiques des diables criminels :

« Alors les diables à cheval arrivent au bord du fleuve, ils ont la peau bleue, de grandes robes noires, ils sont armés de sabres et de lances, ils tuent tout le monde dans le village et ils emmènent les enfants, loin, très loin à travers la forêt et le désert, ils galopent dans les herbes, et les enfants sont attachés sur la selle des chevaux comme des moutons qu'on va égorger, ils crient, ils appellent, mais personne ne les entend, les diables les emmènent jusqu'à la mer. ") (Le Clézio, p.88.)

Le Clezio reconnaît la présence des personnages cruels «les diables à cheval». Cette cruauté consiste à donne forme à

12- Télérama, Arabesques-editions.com, Multi.fi

13. «Danser l'écriture comme on dit danser le tango: c'est faire d'emblée l'hypothèse que la danse est la métaphore de l'écriture, que l'écrit est une danse de mots et plus encore une véritable incorporation $\mathrm{du}$ flux corporel/énergétique, plus continu que le terme de « sismographe » qualifiant l'écriture leclézienne ne le laisse supposer. » Le Clézio, danser l'écriture Isabelle Roussel-Gillet, p. 203-216 


\section{مجلة وادي النيل للاراسات والبحوث الإنسانية والاجتماعية والتربوية (مجلة علمية محكمة)}

(ISSN : 2536 - 9555)

une véritable monstruosité esthétique «ils ont la peau bleue». La couleur bleu de leur peau fait de ces diables des personnes extra-supérieures. Le thème de la monstruosité est exploité dans Alma qui nous apprend les exactions commises sur «tout le monde dans le village». Les diables bleus, qui parcourent le village à cheval, colonisent pour toujours cette petite géographie mauricienne.

Notre romancier s'efforce ensuite de décrire à travers les diables à cheval l'exemple des «barbares» qui cherchent à se définir et à se former des personnalités criminelles : «ils galopent dans les herbes, et les enfants sont attachés sur la selle des chevaux comme des moutons qu'on va égorger, ils crient, ils appellent, mais personne ne les entend, les diables les emmènent jusqu'à la mer. »

Les diables à cheval ne sont-ils pas la pire des barbaries. Priver les enfants et les femmes de leur liberté n'est-elle pas la pire des vengeances : n'est-ce pas le caractère de ce qui est propre à l'animalité. ${ }^{(14)}$ Examinons le passage suivant :

«Ensuite, Topsie, raconte ! Ensuite, dit Topsie, il fait très chaud dans le ventre du bateau, et ça pue le caca et le pipi, et aussi l'odeur du sang des femmes, alors pour laver le bateau les diables noirs jettent des seaux d'eau de mer, ils ne donnent à manger qu'une seule fois par jour, de la pâte d'igname et une calebasse d'eau, et les enfants se battent entre eux pour voler la nourriture et l'eau. Qu'est-ce qui est arrivé, qu'est-ce qu'il s'est passé après ? Raconte, Topsie, raconte ! Après, dit Topsie, le bateau arrive dans une grande île où les

14 - «Nous devons nous élever de toutes nos forces et inlassablement contre les envahissements de toutes les barbaries. » Pensées de Charles Péguy. 
L'imaginaire géographique dans Alma de Jean-Marie Le Clézio Étude géocritique

Dr. Kamel Mounir

مجلة وادي النيل للاراسات والبحوث الإنسانية والاجتماعية والتريوية (مجلة علمية محكمة)

habitants ne sont pas noirs, ni arabes, ils sont petits et jaunes, et les diables ont emmené les femmes et les enfants sur l'île et j'ai pensé qu'ils les emmenaient pour les manger, et le nom de cette île je m'en souviens c'était Mafia. » (Le Clézio, pp. 89-90.)

S'il fallait une image réelle pour résumer la souffrance qui s'est abattue sur cette petite géographie maritime, ce serait celle-ci : «ils ne donnent à manger qu'une seule fois par jour, de la pâte d'igname et une calebasse d'eau, et les enfants se battent entre eux pour voler la nourriture et l'eau. »

Il convient de rappeler que la discussion autour de la barbarie dans Alma semble déboucher sur une critique de fond, sur laquelle Le Clézio en conclut à des idées qui émergent en cheminant. Les critiques sur la tentative de barbares de coloniser le territoire mauricien ont pour origine le fait qu'il écrit : « les diables armés de tromblons et de gourdins les tueraient par centaines, jusqu'à ce qu'il n'en reste que des os. Un monde où les plages de sable blanc seraient bientôt semées de petites boules gluantes et noires, où les vagues venues de l'autre bout du monde apporteraient leurs lots de sacs en plastique et de vieilles bouteilles. Ou bien peut-être qu'il n'a rien compris, rien imaginé, et que la nature sans pitié a fait le reste. » (Le Clézio, p. 31.)

Le déchaînement de la terreur qui s'est abattue sur cette région du monde atteint tous, d'une manière qui dépasse même souvent ce que l'on peut comprendre: « Ou bien peut-être qu'il n'a rien compris, rien imaginé, et que la nature sans pitié a fait le reste. » 
مجلة وادي النيل للار اسات والبحوث الإنسانية والاجتماعية والتربوية (مجلة علمية محكمة)

(ISSN : 2536 - 9555)

\section{Conclusion}

$\mathrm{Au}$ terme de notre article sur l'imaginaire géographique dans Alma de Jean-Marie Le Clézio, nous avons mieux découvert des principales thématiques dont la portée dépasse le cadre de cet article. La géocritique appliquée sur Alma de Le Clézio aborde notamment l'espace comme un champ d'expérience. Dans son plus récent roman, Alma, nous remarquons chez Le Clézio un désir d'appuyer son goût pour le choix de nom lieu à référence géographique. Cela peut servir à donner une définition de l'écriture leclézienne qui semble former un cadre historique de la littérature coloniale. Il fallait donc jongler entre l'acte de barbarie et la centralité géographique. Il nous semble opportun de citer Caligaris: "L'acte de barbarie ne se caractérise pas exactement par l'annulation de toute valeur morale, mais plutôt par la redistribution des valeurs morales sur l'échelle qui les hiérarchise. "(Caligaris 2013 : 86). Le monde de Le Clézio a ses lois : il aborde des jugements sociaux et des relations à la fois humaines et inhumaines.

\section{RÉFÉRENCES BIBLIOGRAPHIQUES}

\section{Corpus}

J. M. G. LE CLÉZIO, (1917), Alma, paris, Gallimard, Collection «Blanche».

\section{Ouvrages consultés}

-Labbé Michelle, (1999), L'Écart romanesque, paris, Harmattan.

-Caligaris, Nicole (2013). Le paradis entre les jambes. Paris, Verticales.

-Maurice Blanchot, L'espace littéraire, Paris, Gallimard, Folio, essais, 1955, 2007. 


\section{L'imaginaire géographique dans Alma de Jean-Marie Le Clézio Étude géocritique}

Dr. Kamel Mounir

$$
\text { مجلة وادي النيل للاراسات والبحوث الإنسانية والاجتماعية والتربوية (مجلة علمية محكمة) }
$$

-Gérard de Cortanze, J.-M. G. Le Clézio, le nomade immobile, Paris, Éditions du chêne, 1999.

-Bertrand Westphal, Réel, fiction, espace, Paris, Éditions de minuit, 2007.

-Michel Roux, Géographie et complexité. Les espaces de la nostalgie, Paris, Montréal, Éditions, Harmattan, 1999.

\section{Webographie}

https://www.literature.green/problematiques-ecologiques-dans-lesecrits-de-jean-marie-gustave-le-clezio-universite-de-dschangcameroun/

https://books.openedition.org/pur/40289

https:/www.cairn.info/revue-espace-geographique-2016-4-page289.htm

https://sflgc.org/bibliotheque/westphal-bertrand-pour-uneapproche-geocritique-des-textes/

http://ilemauricehotels.com/ile-maurice-histoire.html https://www.franceculture.fr/emissions/le-cours-de-1-histoire/lecours-de-1-histoire-emission-du-jeudi-09-septembre-2021

https://bibliobs.nouvelobs.com/actualites/20180322.OBS4044/disp arition-des-oiseaux-les-ecrivains-1-avaient-predit.html

https://czasopisma.bg.ug.edu.pl/index.php/CE/article/view/3922

https://www.enviedecrire.com/lecriture-selon-jean-marie-gustavele-clezio/

https://books.openedition.org/pur/40315

https://almaconsult-paris.com/inspiration/le-tambalacoque-almaconsult-aime-alma-de-jmg-clezio.html 
مجلة وادي النيل للاراسات و البحوث الإنسانية والاجتماعية والتربوية (مجلة علمية محكمة)

(ISSN : 2536 - 9555)

http://evene.lefigaro.fr/citation/devoir-aimer-langue-maternellenegliger-signe-decadence-morale-59728.php

https://www.dicocitations.com/citation.php?verif_robot $=\&$ motcle $=$ barbaries\&base $=1$ 